\title{
A Hybrid Adaptive Control Strategy for a Smart Prosthetic Hand
}

\author{
Cheng-Hung Chen, Student Member, IEEE, D. Subbaram Naidu, Fellow, IEEE, Alba Perez-Gracia, and \\ Marco P. Schoen, Member, IEEE
}

\begin{abstract}
This paper presents a hybrid of a soft computing technique of adaptive neuro-fuzzy inference system (ANFIS) and a hard computing technique of adaptive control for a twodimensional movement of a prosthetic hand with a thumb and index finger. In particular, ANFIS is used for inverse kinematics, and the adaptive control is used for linearized dynamics to minimize tracking error. The simulations of this hybrid controller, when compared with the proportional-integral-derivative (PID) controller showed enhanced performance. Work is in progress to extend this methodology to a five-fingered, three-dimensional prosthetic hand.
\end{abstract}

Index Terms - Prosthetic Hand, Adaptive Control, PID Control, Adaptive Neuro-Fuzzy Inference System, Genetic Algorithm, Hybrid Control

\section{INTRODUCTION}

Artificial hands have been around for the last several years developed by various researchers in the field [1]-[3]. Yet, about $35 \%$ of the amputees do not use their prosthetic hand regularly due to various reasons such as poor functionality of the presently available prosthetic hands and psychological problems. To overcome this problem, one has to design and develop an artificial hand which "mimics the human hand as closely as possible" both in functionality and appearance.

Soft computing (SC) or computational intelligence (CI) [4] is an emerging field based on synergy and seamless integration of neural networks (NN), fuzzy logic (FL) and genetic algorithms (GA). The previous works on prosthetic hand used artificial NN, FL, GA etc. mostly for EMG signal classification for various movements or functions of the prosthetic hand. Hard control (HC) techniques comprise proportional-integral-derivative (PID) control [5]-[7], optimal control [8], adaptive control [9], [10] etc. with specific applications to prosthetic devices. However, our previous works [6], [7] for smart prosthetic hand showed that PID controller results in overshooting and oscillation. In addition, the main challenge to improve the prosthetic hand performance is to estimate the disturbances, including

Cheng-Hung Chen is with the Measurement and Control Engineering Research Center and the Department of Biological Sciences, Idaho State University, ID 83209, USA (email: chenchen@isu.edu).

D. Subbaram Naidu is with the Measurement and Control Engineering Research Center and the Department of Electrical Engineering, Idaho State University, ID 83209, USA (email: naiduds@isu.edu).

Alba Perez-Gracia is with the Measurement and Control Engineering Research Center and the Department of Mechanical Engineering, Idaho State University, ID 83209, USA on leave with Institut de Robotica i Informatica Industrial (UPC-CSIC) c/ Llorens i Artigas 4-6, Barcelona, Spain (email: perealba@isu.edu).

Marco P. Schoen is with the Measurement and Control Engineering Research Center and the Department of Mechanical Engineering, Idaho State University, ID 83209, USA (email: schomarc@isu.edu). internal disturbances and external disturbances [11]. The internal disturbances includes un-modeled dynamics, such as the coupling flexibility in links/joints, and uncertainties, such as unknown parameters. The external disturbances include external forces/moments on the fingertips and friction in joints. To overcome the overshooting and disturbance problems, using adaptive control strategy to prosthetic hand is precisely the main goal of this work.

In this paper, we first consider briefly the trajectory planning and the inverse kinematics for a two-dimensional movement of a two-link thumb and three-link index finger using adaptive neuro-fuzzy inference system (ANFIS) and genetic algorithm (GA). Next, the dynamics of the hand is derived using regression matrix. Then the adaptive controller is designed to reduce the effect of disturbances and unknown parameters, including masses and inertia. The resulting overall hybrid system incorporating both soft and hard control techniques is simulated with practical data for the hand. The last section details the conclusions and future work.

\section{Modeling}

\section{A. Trajectory Planning}

It is necessary to know the desired paths before the prosthetic hand is controlled in order to execute a specific hand motion task. Trajectory planning involves the generation of the desired paths or reference inputs, so trajectory planning is associated with some situations, such as avoiding barriers and making sure that the desired path does not exceed the voltage and torque limitations of the actuators. To generate smooth trajectories, a cubic polynomial function for the fingertip space is created [3], [6], [7].

\section{B. Inverse Kinematics}

Using inverse kinematics, the angular positions (or called joint angles) of each finger can be obtained from the known fingertip positions. A desired trajectory is usually specified in Cartesian space and the trajectory control is easily performed in the joint space. Hence, it is necessary to convert Cartesian trajectory to the joint space [12]-[14].

1) Two-Link Thumb: As shown in Figure 1, thumb is assumed as the two-link finger and the other four fingers, such as index finger, middle finger, ring finger, and little finger, are considered as three-link fingers. Hence, the joint angles, angular velocities, and angular accelerations of each finger can be deduced as follows. According to forward kinematics [12]-[14], the fingertip coordinate $\left(X^{t}, Y^{t}\right)$ of 


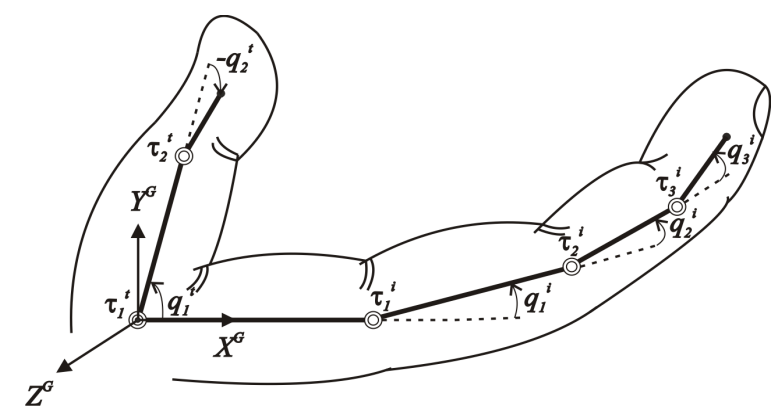

Fig. 1. Schematic Diagram of Thumb and Index Finger

thumb $(t)$ can be described as

$$
\begin{aligned}
& X^{t}=L_{1}^{t} \cos \left(q_{1}^{t}\right)+L_{2}^{t} \cos \left(q_{1}^{t}+q_{2}^{t}\right), \\
& Y^{t}=L_{1}^{t} \sin \left(q_{1}^{t}\right)+L_{2}^{t} \sin \left(q_{1}^{t}+q_{2}^{t}\right) .
\end{aligned}
$$

Here $L_{1}^{t}$ and $L_{2}^{t}$ are the lengths of the link 1 and 2 of thumb, respectively; $q_{1}^{t}$ and $q_{2}^{t}$ are the angles of joint 1 and 2 of thumb. Choosing the elbow up configuration, the angle $q_{2}^{t}$ of the joint 2 can be obtained as:

$$
q_{2}^{t}=-\cos ^{-1}\left(\frac{X^{t^{2}}+Y^{t^{2}}-L_{1}^{t^{2}}-L_{2}^{t^{2}}}{2 L_{1}^{t} L_{2}^{t}}\right) .
$$

Based on the geometry, we can get

$$
q_{1}^{t}=\tan ^{-1}\left(\frac{Y^{t}}{X^{t}}\right)-\tan ^{-1}\left(\frac{L_{2}^{t} \sin \left(q_{2}^{t}\right)}{L_{1}^{t}+L_{2}^{t} \cos \left(q_{2}^{t}\right)}\right) .
$$

Then using differential kinematics, the angular velocities and accelerations of each finger can be obtained from the known fingertip linear velocities and accelerations. The corresponding linear velocities $d\left(X^{t}, Y^{t}\right) / d t$ of the fingertip are obtained as

$$
\dot{\mathbf{P}}^{\mathrm{t}}=\mathbf{J}^{\mathrm{t}} \dot{\mathbf{q}}^{\mathrm{t}}
$$

where, matrices $\dot{\mathbf{P}}^{\mathrm{t}}$ and $\dot{\mathbf{q}}^{\mathrm{t}}$ are

$$
\dot{\mathbf{P}}^{\mathbf{t}}=\left[\begin{array}{c}
\dot{X}^{t} \\
\dot{Y}^{t}
\end{array}\right], \dot{\mathbf{q}}^{\mathbf{t}}=\left[\begin{array}{c}
\dot{q}_{1}^{t} \\
\dot{q}_{2}^{t}
\end{array}\right]
$$

The matrix $\mathbf{J}^{\mathbf{t}}$ is called the Jacobian of thumb and the angular velocities $\dot{q}_{1}^{t}$ and $\dot{q}_{2}^{t}$ of the joint 1 and 2 are

$$
\dot{\mathbf{q}}^{\mathbf{t}}=\mathbf{J}^{\mathbf{t}-1} \dot{\mathbf{P}}^{\mathbf{t}} .
$$

Similarly, the angular accelerations $\ddot{q}_{1}^{t}$ and $\ddot{q}_{2}^{t}$ of the joints 1 and 2 are obtained as

$$
\ddot{\mathbf{q}}^{\mathbf{t}}=\mathbf{J}^{\mathbf{t}^{-1}}\left(\ddot{\mathbf{P}}^{\mathbf{t}}-\frac{d \mathbf{J}^{\mathbf{t}}}{d \mathbf{t}} \dot{\mathbf{q}}^{\mathbf{t}}\right)
$$

where $\ddot{\mathbf{P}}^{t}$ is the acceleration vector of the fingertip. $\ddot{\mathbf{P}}^{t}$ and $\ddot{\mathbf{q}}^{\mathrm{t}}$ are denoted as

$$
\ddot{\mathbf{P}}^{\mathbf{t}}=\left[\begin{array}{c}
\ddot{X}^{t} \\
\ddot{Y}^{t}
\end{array}\right], \ddot{\mathbf{q}}^{\mathbf{t}}=\left[\begin{array}{c}
\ddot{q}_{1}^{t} \\
\ddot{q}_{2}^{t}
\end{array}\right] .
$$

Similarly for three-link index finger, the fingertip Cartesian coordinates $\left(X^{i}, Y^{i}\right)$ are described in terms of three joint variables $q_{1}^{i}, q_{2}^{i}$, and $q_{3}^{i}$ [3], [7].

\section{Dynamics of Hand}

It is necessary to have a mathematical model that describes the dynamic behavior of prosthetic hand for the purpose of designing the control system. The dynamic equations of hand motion are derived via Lagrangian approach using kinetic energy and potential energy as [10], [14]

$$
\mathbf{M}(\mathbf{q}) \ddot{\mathbf{q}}+\mathbf{C}(\mathbf{q}, \dot{\mathbf{q}})+\mathbf{G}(\mathbf{q})+\boldsymbol{\tau}_{d i s}=\boldsymbol{\tau},
$$

where $\mathbf{M}(\mathbf{q})$ is the inertia matrix; $\mathbf{C}(\mathbf{q}, \dot{\mathbf{q}})$ is the Coriolis/centripetal vector and $\mathbf{G}(\mathbf{q})$ is the gravity vector; $\boldsymbol{\tau}_{\text {dis }}$ is the disturbance vector. Eq. (10) can be also written as

$$
\mathbf{M}(\mathbf{q}) \ddot{\mathbf{q}}+\mathbf{N}(\mathbf{q}, \dot{\mathbf{q}})=\tau,
$$

where $\mathbf{N}(\mathbf{q}, \dot{\mathbf{q}})=\mathbf{C}(\mathbf{q}, \dot{\mathbf{q}})+\mathbf{G}(\mathbf{q})+\boldsymbol{\tau}_{\text {dis }}$ represents nonlinear terms. The dynamic relations for the two-link thumb and the three-link index finger are quite lengthy [3], [11], [15], [16].

\section{Hybrid Adaptive Control Technique}

The tracking error $\mathbf{e}$ and the filtered tracking error $\mathbf{r}$ are defined as

$$
\begin{aligned}
& \mathbf{e}=\mathbf{q}_{\mathbf{d}}-\mathbf{q}, \\
& \mathbf{r}=\dot{\mathbf{e}}+\Lambda \mathbf{e} .
\end{aligned}
$$

Here, $\mathbf{q}_{\mathbf{d}}$ is the desired angle vector of joints and can be obtained by (3) and (4); q is the actual angle vector of joints; $\boldsymbol{\Lambda}$ is the positive-definite diagonal gain matrix. The filtered error (13) ensures stability of the overall system so that the tracking error (12) is bounded. Figure 2 shows the block diagram of the hybrid adaptive controller. Here, the filtered signal $\mathbf{r}(t)$ is derived from the tracking error $\mathbf{e}(t)$ and the ANFIS based trajectory planner and is fed to the adaptive controller of the prosthetic hand.

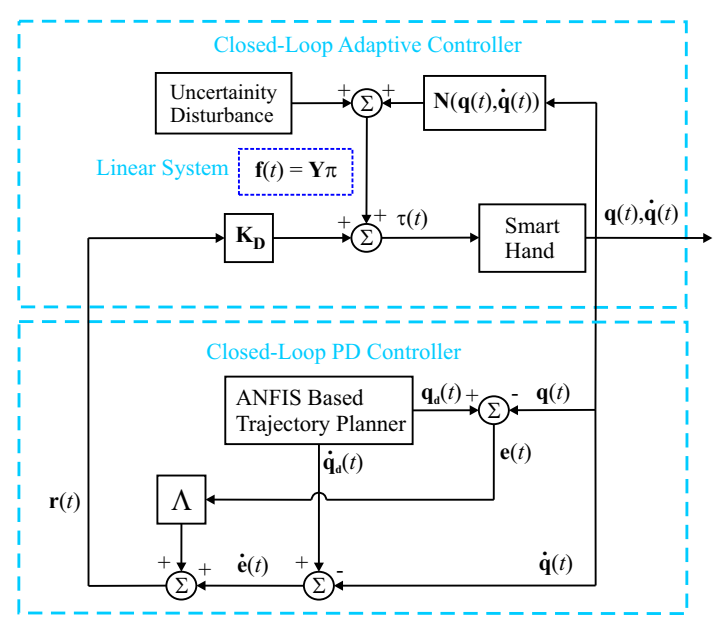

Fig. 2. Block Diagram of Hybrid Adaptive Controller

Differentiating and substituting (13) into (10) gives the dynamic equation in terms of the filtered error $\mathbf{r}$ as

$$
\mathbf{M}(\mathbf{q}) \dot{\mathbf{r}}=-\mathbf{C}_{m}(\mathbf{q}, \dot{\mathbf{q}}) \mathbf{r}+\mathbf{f}-\boldsymbol{\tau}
$$


where $\mathbf{C}(\mathbf{q}, \dot{\mathbf{q}})=\mathbf{C}_{m}(\mathbf{q}, \dot{\mathbf{q}}) \dot{\mathbf{q}}$ and the nonlinear term $\mathbf{f}$ can be defined as

$$
\begin{aligned}
\mathbf{f}= & \mathbf{M}(\mathbf{q})\left(\ddot{\mathbf{q}}_{d}+\mathbf{\Lambda} \dot{\mathbf{e}}\right)+\mathbf{C}_{m}(\mathbf{q}, \dot{\mathbf{q}})\left(\dot{\mathbf{q}}_{d}+\mathbf{\Lambda} \mathbf{e}\right) \\
& +\mathbf{G}(\mathbf{q})+\boldsymbol{\tau}_{d i s} \\
= & \mathbf{Y} \boldsymbol{\pi} .
\end{aligned}
$$

Here, the regression matrix $\mathbf{Y}$ is a matrix of known robot functions and $\boldsymbol{\pi}$ is a vector of unknown parameters [9]. The torque vector $\tau$ can be calculated by

$$
\tau=\mathbf{Y} \boldsymbol{\pi}+\mathbf{K}_{\mathrm{D}} \mathbf{r}
$$

The unknown parameter rate vector $\dot{\pi}$ can be updated by

$$
\dot{\pi}=\Gamma^{-1} \mathbf{Y}^{\prime} \mathbf{r}
$$

where $\Gamma$ is a tuning parameter diagonal matrix.

\section{Simulation Results And Discussion}

Figure 3 shows the forward kinematics (upper row) in terms of the thumb link angles and the tip positions and the inverse kinematics (lower row) of the thumb in terms of the tip positions and the link angles. Figure 4 shows forward
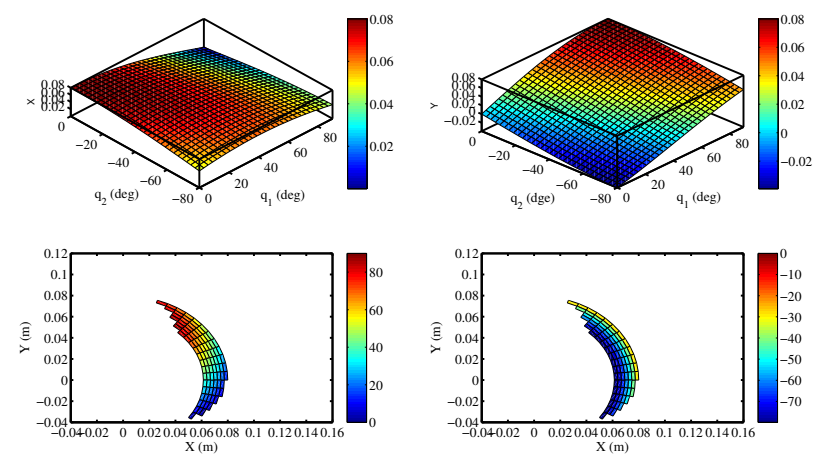

Fig. 3. Forward Kinematics and Inverse Kinematics of Thumb

kinematics (upper row) in terms of the index finger link angles and the tip positions and inverse kinematics (lower row) in terms of the tip positions and the link angles of the index finger by using ANFIS and similar results were obtained using GA. During our simulations [7], we found that the GA method although give a better solution (error $\approx 10^{-7}$ ), takes more execution time whereas the ANFIS gave a good solution (error $\approx 10^{-4}$ ) with less time compared to GA method.

When thumb and fingers are doing extension/flexion movements, the ranges that fingertips can reach are restricted to the reach of the angles of the joints. Referring to inverse kinematics, Figure 5 explains that the movable range of the fingertip of the thumb, in which the first and second joint angles are constrained in the ranges of $[0,90]$ and $[-80,0]$ (degrees). The first, second, and third joint angles of the index finger are constrained in the ranges of $[0,90],[0,110]$ and [0,80] (degrees), respectively [17]. Thus, the green and pink regions respectively show the reachable fingertip positions of thumb and index finger. Further, the overlap
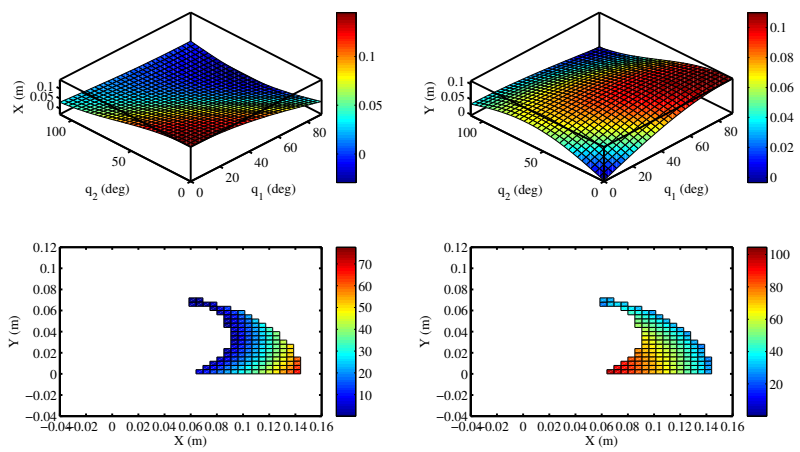

Fig. 4. Forward Kinematics and Inverse Kinematics of Index Finger Using ANFIS

region represents that the space that both the thumb and the index finger can reach.

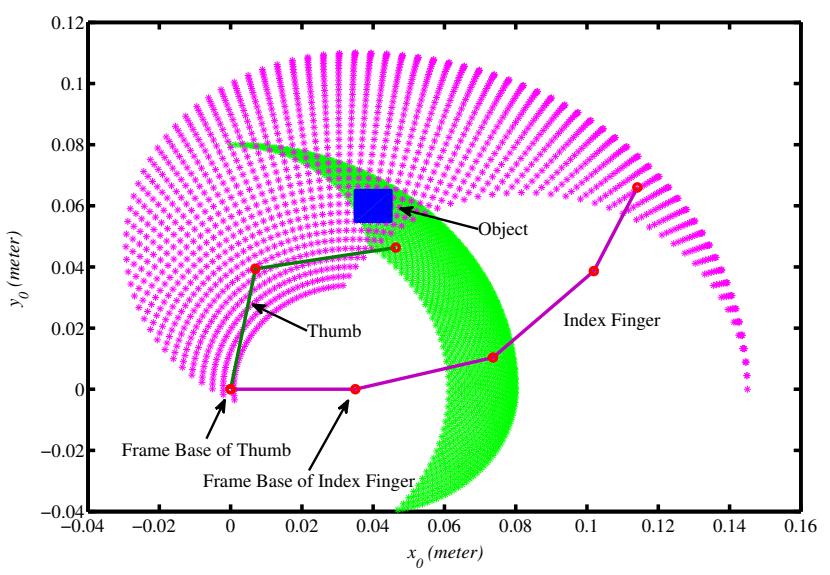

Fig. 5. The Movable Range of the Fingertips of Thumb and Index Finger and Square Object

Next, we present simulations with a PID controller and hybrid adaptive controller for the two-link thumb and threelink index finger of a smart prosthetic hand. To demonstrate the accuracy of the proposed hybrid adaptive controller, we present two desired paths. The desired path 1 for two-link thumb is cubic polynomial function. The desired path 2 for three-link index finger is

$$
\mathbf{q}_{d}^{i}=\left[A_{1}^{i} \sin \left(\frac{2 \pi}{T}\right) \quad A_{2}^{i} \sin \left(\frac{2 \pi}{T}\right) \quad 0.7 A_{2}^{i} \sin \left(\frac{2 \pi}{T}\right)\right]^{\prime} .
$$

The various parameters [18] relating to desired trajectory and the two-link thumb/three-link index finger selected for the simulations are given as: $A_{1}^{i}=A_{2}^{i}=0.1 ; T=2$ (path 2); initial position $\left(X^{t}, Y^{t}\right)=(0.035,0.060)$ and final position $\left(X^{t}, Y^{t}\right)=(0.0495,0.060)(\mathrm{m})$; initial and final velocities are zero (path 1); the lengths of the links 1,2 and 3 are $0.040,0.040$, and $0.030(\mathrm{~m})$; simulation time for paths 1,2 and 3 is 10,10 and 20 seconds, respectively. PID diagonal coefficients, $\mathbf{K}_{\mathrm{p}}, \mathbf{K}_{\mathbf{i}}$ and $\mathbf{K}_{\mathbf{d}}$, are 100 . As for hybrid adaptive diagonal coefficients, $\mathbf{K}_{\mathbf{D}}, \boldsymbol{\Lambda}$, and $\boldsymbol{\Gamma}$ are chosen as 100 . 
Figures 6 and 7 show that our previous work [6], [7] with PID controller and the presented hybrid adaptive control method for two-link thumb and three-link index finger. It is clearly seen that the results using proposed hybrid adaptive control method can overcome the overshooting and oscillation problems even if the prosthetic hand is under disturbances.
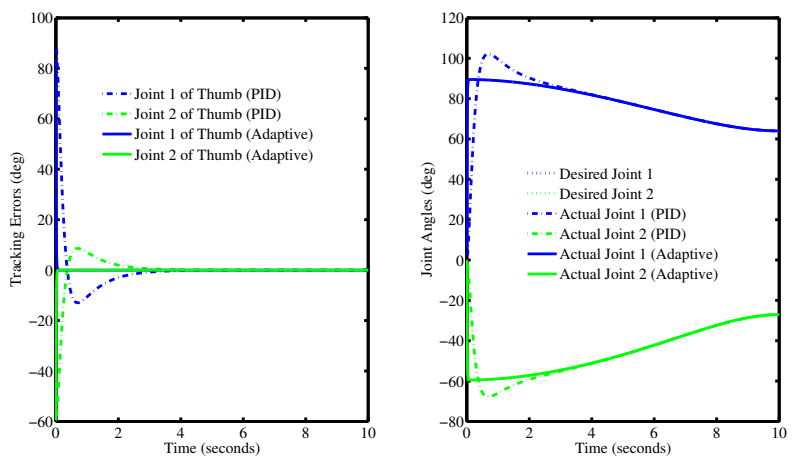

Fig. 6. Tracking Error (left) and Joint Angle (right) for PID and Hybrid Adaptive Controllers of Thumb for Path 2
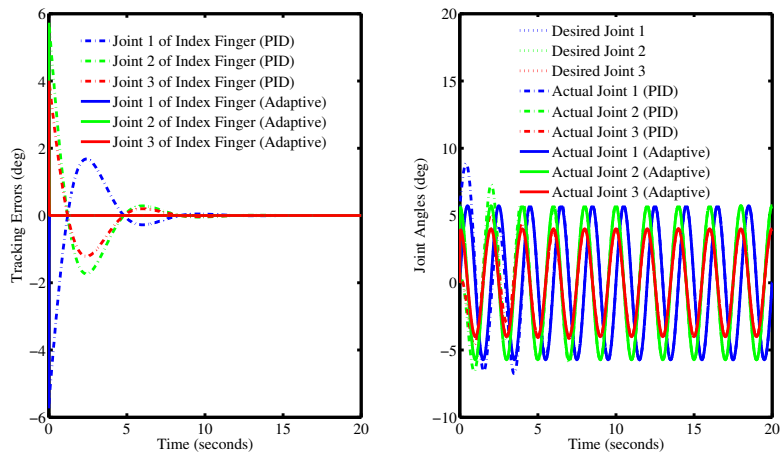

Fig. 7. Tracking Error (left) and Joint Angle (right) for PID and Hybrid Adaptive Controllers of Index Finger for Path 3

\section{CONCLUSions And Future Work}

This paper focussed on hybrid of soft computing technique of adaptive neuro-fuzzy inference system (ANFIS) and hard control technique of adaptive control for a two-dimensional movement with thumb and index prosthetic hand. In particular, ANFIS was used for inverse kinematics, and the dynamics of the hand is derived using regression matrix. An adaptive control strategy was applied to the prosthetic hand to reduce the effect of disturbances and unknown parameters, including masses and inertia. The resulting overall hybrid system incorporating both soft and hard control techniques is simulated with practical data for the hand. The simulations using this hybrid controller, when compared with the proportional-integral-derivative (PID) controller showed enhanced performance. Work is underway to extend this methodology to a five-fingered, three-dimensional prosthetic hand based on the works [19], [20].

\section{ACKNOWLEDGMENTS}

The financial support for this research from the Telemedicine Advanced Technology Research Center (TATRC) of the US Department of Defense (DoD) is acknowledged.

\section{REFERENCES}

[1] L. Zollo, S. Roccella, E. Guglielmelli, M. C. Carrozza, and P. Dario, "Biomechatronic design and control of an anthropomorphic artificial hand for prosthetic and robotic applications," IEEE/ASME Transactions on Mechatronics, vol. 12, no. 4, pp. 418-429, August 2007.

[2] D. S. Naidu, C.-H. Chen, A. Perez, and M. P. Schoen, "Control strategies for smart prosthetic hand technology: An overview," in Proceedings of the 30th Annual International IEEE EMBS Conference, Vancouver, Canada, August 20-24 2008, pp. 4314-4317.

[3] C.-H. Chen, "Hybrid control strategies for smart prosthetic hand," Ph.D. dissertation, Measurement and Control Engineering, Idaho State University, May 2009.

[4] A. Konar, Computational Intelligence: Principles, Techniques and Applications. Berlin, Germany: Springer-Verlag, 2005.

[5] B. Subudhi and A. S. Morris, "Soft computing methods applied to the control of a flexible robot manipulator," Applied Soft Computing, vol. 9, pp. 149-158, 2009.

[6] C.-H. Chen, K. W. Bosworth, M. P. Schoen, S. E. Bearden, D. S. Naidu, and A. Perez, "A study of particle swarm optimization on leukocyte adhesion molecules and control strategies for smart prosthetic hand," in 2008 IEEE Swarm Intelligence Symposium (IEEE SIS08), St. Louis, Missouri, USA, September 21-23 2008.

[7] C.-H. Chen, D. S. Naidu, A. Perez, and M. P. Schoen, "Fusion of hard and soft control techniques for prosthetic hand," in Proceedings of the International Association of Science and Technology for Development (IASTED) International Conference on Intelligent Systems and Control (ISC 2008), Orlando, Florida, USA, November 16-18 2008, pp. 120125.

[8] D. Naidu, Optimal Control Systems. Boca Raton, FL: CRC Press, 2003.

[9] F. L. Lewis, S. Jagannathan, and A. Yesildirek, Neural Network Control of Robotic Manipulators and Nonlinear Systems. London, UK: Taylor \& Francis, 1999.

[10] F. L. Lewis, D. M. Dawson, and C. T. Abdallah, Robot Manipulators Control: Second Edition, Revised and Expanded. New York, NY: Marcel Dekker, Inc.,, 2004.

[11] A. Nikoobin and R. Haghighi, "Lyapunov-based nonlinear disturbance observer for serial n-link robot manipulators," Journal of Intelligent and Robotic Systems, 2008, (Published online on 11 December 2008).

[12] R. Kelly, V. Santibanez, and A. Loria, Control of Robot Manipulators in Joint Space. New York, USA: Springer, 2005.

[13] R. N. Jazar, Theory of Applied Robotics. Kinematics, Dynamics, and Control. New York, USA: Springer, 2007.

[14] B. Siciliano, L. Sciavicco, L. Villani, and G. Oriolo, Robotics: Modelling, Planning and Control. London, UK: Springer-Verlag, 2009.

[15] Y. Z. Arslan, Y. Hacioglu, and N. Yagiz, "Prosthetic hand finger control using fuzzy sliding modes," Journal of Intelligent and Robotic Systems, vol. 52, pp. 121-138, 2008.

[16] C.-H. Chen and D. Naidu, "Hybrid control strategies for prosthetic hand," Measurement and Control Engineering Research Center, Idaho State University, Pocatello, ID, Tech. Rep., July 2008.

[17] P. K. Lavangie and C. C. Norkin, Joint Structure and Function: A Comprehensive Analysis, Third Edition. Philadelphia, PA: F. A. Davis Company, 2001.

[18] S. Arimoto, Control Theory of Multi-fingered Hands: A Modeling and Analytical-Mechanics Approach for Dexterity and Intelligence. London, UK: Springer-Verlag, 2008.

[19] D. G. Kamper, E. G. Cruz, and M. P. Siegel, "Stereotypical fingertip trajectories during grasp," J. Neurophysiol., vol. 90, pp. 3702-3710, 2003.

[20] E. Cruz, H. Waldinger, and D. Kamper, "Kinetic and kinematic workspaces of the index finger following stroke," Brain, vol. 128, pp. 1112-1121, 2005. 